\title{
Working Memory Deficits in Depression and Related Interventions
}

\author{
Rouchi Guo ${ }^{1, *, \dagger} \mathrm{Jiaxi}^{\mathrm{Li}^{2, *}, \dagger}$ \\ ${ }^{1}$ University of Pennsylvania, Philadelphia, PA, 19104, USA \\ ${ }^{2}$ Bukkyo University, Kyoto, Kyoto, 603-8301, Japan \\ *Corresponding author.Email: 'rouchigu@upenn.edu., ${ }^{*}$ lijiaxi123@gmail.com \\ Those authors contributed equally.
}

\begin{abstract}
Working memory is short-term recognition memory processed by the prefrontal cortex, which may play an important role in reasoning, decision making, and the guidance of behaviour. It is difficult for people with depression to make the decision and achieve goals. Thereby, it is significant to learn working memory defects in depression and to develop related interventions. Attachment patterns, the social competition hypothesis, and the hopelessness theory of depression have been extensively studied. It is suggested that early childhood environment and personal encounter may be the two most important factors for the formation of depression. As the defects and functions of working memory are still quite limited, this review discussed the relationship between working memory and depression from the following three aspects: the update of emotion-related working memory in depression, the neural mechanism, and related intervention for people with depression. One of the limitations of existing studies is that they focused on cross-sectional comparisons. Future research should conduct longitudinal studies. Another is that many tasks are cognitive tasks. However, the renewal of working memory in real life and natural environment is very complicated. Most of the researchers should rely on many life taskings. In addition, existing studies regarding working memory defect as a single factor, without considering its relationship with other factors. Therefore, future studies can investigate more on the impact of working memory defects on depression.
\end{abstract}

Keywords: working memory, deficits, depression, neural mechanism, interventions.

\section{INTRODUCTION}

Unlike the storage functions of long-term and shortterm memory, working memory refers to goal-directed behaviours, and information must be retained and manipulated to successfully execute a task [1]. Therefore, working memory is significant for reasoning, decisionmaking, and the guidance of behaviour. If people don't have an intact working memory, have problems with working memory, people will not be able to complete some of the learning tasks. For example, when people are given instructions for complicated appliances, they may not keep all the steps in mind or mess up the correct order. Another situation is that if a teacher asks a student to do $16+25$ in their heads, the student may not immediately work out the sum of 41 or even forget what the teacher asked them to do with the two numbers. Depression is a type of mood regulation disorder, and the main characteristic of depression is the decrease of positive emotion and persistent negative emotion [2]. Similarly, people with depression have difficulties making decisions and achieving goals in life and study, and working memory is closely related to achieving goals. Because of the importance of working memory, if researchers can have a deeper understanding of the working memory of depression, they will find a more comprehensive treatment for depression.

One of the major theories of depression is the attachment theory proposed by Bowlby. According to Bowlby, attachment is initially the affectional bonds or attachments between a child and a parent and later between adult and adult in the process of healthy development [3]. He considered that sadness is also presented in the attachment patterns of infants and their primary caregivers, usually the mother. For example, the anxiety and sadness that infants exhibit when they are separated from their mothers is a reaction to temporary loss. It can help re-establish physical intimacy. Furthermore, Bowlby points out that MDD is a 
maladaptive grieving or mourning caused by distorted perceptions of the self that are rooted in childhood attachment problems, such as childhood distress, physical and sexual abuse, parental mental illness, all of which have been connected to adult mood disorders to some extent. Another major theory is the social competition hypothesis which suggests that depression is a submissive emotion, a dysfunctional involuntary subordination strategy [3]. When in a weak position in social competition, this subjective sense of incompetence can refrain aggression on higher-ranked people and advance concessions by exhibiting submissive behaviour to minimize losses [4]. The social competition hypothesis is also consistent with the hopelessness theory of depression. This theory predicts that the interaction between negative cognitive patterns and negative life events will generate hopelessness and postulates that such feelings of hopelessness will lead to depression [5].

Most of the current work on depression focuses on the developmental perspective of its prevalence, symptoms, and etiology, suggesting early childhood environment and personal encounters maybe two of the most important contributors to the formation of depression. Moreover, working memory, the short-term recognition memory that processes at the brain's prefrontal cortex, is a pivotal indicator of an individual's cognitive performance and capacity. It is associated with decisionmaking, recognition, and other cognitive functions. However, neurological and psychiatric R\&D on MD in terms of impairment, capacity, and functionality of working memory is still fairly limited nowadays. This lends credence to the reason for our review. Our literature review on working memory and its relation to depression symptoms from psychiatric and neurological perspectives fill in the gap between cognitive psychology and psychiatry on MD treatment and intervention. We discussed the relationship between working memory deficits and depression from three aspects. First, emotion-related working memory updating in depression. Second, cognitive neural mechanisms underlying the working memory deficits. Finally, relevant interventions at the level of both behavioural and neural for depression.

\section{EMOTIONAL PROCESSING AND WORKING MEMORY DEFICITS IN DEPRESSION}

The ability to update working memory is an essential part of cognitive and emotional regulation [6]. To deal with problems and regulate emotions, people need to process a large collection of information constantly, so they must update the contents of working memory fluidly, keeping only relevant information. The root cause of emotional regulation problems may be difficulties in updating stimuli in working memory, and emotional problems may also contribute to the development and persistence of depression. Therefore, it is significant to explore whether people with depression have deficits in working memory and how those deficits influence emotional processing. Levens et al. examined the ability to update emotional stimuli in working memory in depressed participants and non-depressed participants [6]. The tasks were demanded participants to complete two tasks: 0-back and 2-back. The 0-back required the participants to look at a happy, sad, or neutral "expression label" and a sample face of the same expression, and if the expression was the same as the target expression, participants needed to press the 'Same' key, and then press a 'Diff' key if the expression was different from the target expressions. The 2-Back asked participants to indicate whether the current expression was the same or different from the previous two expressions and to press the 'Same' or a 'Diff' key. In the 0-back task, both depressed and non-depressed participants were faster at distinguishing happy stimuli than neutral and sad stimuli. In the two-back tasks, depressed participants replace sad stimulation more slowly than non-depressed participants and replace happy stimulation more quickly. In contrast, non-depressed participants took longer to break away from happy stimulation than from sad or neutral stimulation. The results suggest that these intergroup differences in reaction times may reflect maladaptive and protective biases of working memory in depression. These biases may interfere with their ability to effectively regulate negative emotions.

The question of whether updating working memory is easily interfered with by irrelevant negative emotion due to depression symptoms is yet to be fully explored. In the research conducted by Joormann and Gotlib in 2008, they mainly investigated the relation between working memory and depression along with rumination. Intrusion effect is the extent to which irrelevant emotional material can intrude into working memory. Response latencies capture the intrusion effect, and it is measured as the extent of depression symptoms that manifest on updating working memory. In the method section, researchers enacted emotion manipulation by letting participants listen to sad music before the recognition tasks. Then, participants were asked to complete several trials related to memorizing and ignoring emotion-related words with designated instruction. The result suggested that depressed participants were more influenced by the intrusion effect when presented with negative words than participants in other groups. A higher intrusion effect indicates a higher difficulty in removing negative material from working memory, and such difficulty is also associated with self-reported rumination [7].

Furthermore, the relation between inhibition control and the deficit in working memory is also vitally important in terms of researching depression symptoms. Another research from Joormann and Gotlib in 2010 investigated depression and its relation to cognition inhibition, mainly the inhibition of negative emotion materials. The negative affective priming task is the 
adapted evaluation method that assesses the cognitive inhibition and its relation to emotion regulation habits among diagnosed depressed participants when they process rumination, reappraisal, and expressive suppression in emotion [2]. Methods are mainly in questionnaire format, evaluating self-reported rumination and other series of negative emotions, depression symptoms, and general cognitive function. The result suggests a reduction of inhibition among depressed participants when they process those negative emotion materials. The reduction is also associated with higher levels of termination, expressive suppression in emotion, and less use of reappraisal. Therefore, individual differences do play an important role in determining cognitive inhibition, and depression results in deficits in working memory.

\section{NEURAL MECHANISM UNDERLYING THE WORKING MEMORY DEFICITS}

Since it is challenging to update the emotional information, it is important to understand the mechanisms underlying the deficiency in working memory. What's more, it is also significant to perceive the symptoms of depression and to develop more effective interventions. A deficiency in working memory in relation to updating emotions may be responsible for some problems with social activities. In general, in addition to updating emotions, common deficiencies in WM can also be problematic and influence social interaction. Rumination is an unintentional and uncontrollable symptom of depression related to the recurrence of depression [8]. Ruminators tend to dwell on negative events and thoughts and have a hard time turning them into a new thought, which is suggested to be associated with working memory defects. Thus, understanding rumination and deficits in general working memory are vital for understanding depression. Joormann conducted a study on the association between rumination and depression in working memory, in which the ability to maintain or reverse the order of three emotional words in WM was evaluated [9]. The participants were asked to remember words as quickly and accurately as possible. After watching three words at a time on a computer monitor, they were required to remember the words in the order in which they were presented in forwarding trials. Then, in the backward trials, they were required to reverse the order and resort them to WM. The researchers also calculated the sorting costs, the difference between response delays in the forward and backward trials. They found that the depressed group demonstrated a higher sorting cost level when confronting negative words than the control group. Likewise, in the depressed group, rumination did predict the presence of negative words. The results showed that defects in cognitive control are associated with rumination.
Working memory is associated with the activation of the prefrontal cortex. The prefrontal cortex is related to regulating emotional cognition and behaviour control [10]. If patients with depression have problems with their working memory, they may indeed have problems, and it will be very likely to reflect on their relevant neural mechanism. Matsuo et al. used the N-back task to measure working memory function in depression with functional magnetic resonance imaging (fMRI) [11]. The $\mathrm{N}$-back task is a stimulus test, 0-back, 1-back,2-back was performed 5 times, which is commonly used as a working memory task, that can also trigger manipulation of working memory processes such as maintenance, monitoring, updating, and memorizing the information. The results revealed there are no significant differences in working memory accuracy or response time between MDD patients and healthy individuals. Still, MDD patients had higher activation in the dorsolateral prefrontal cortex (DLPFC) than controls. Since there is no difference in behaviour, it helps us to understand how they differ in neural mechanisms when they behave in the same way. Moreover, understanding these differences will allow us to better grasp the variation that might arise when faced with more challenging tasks. The findings indicated that working memory defects in depression are associated with an overactive prefrontal cortex, which might reflect a tendency to think too much, be oversensitive or be overactive.

Studies have shown that working memory-related neural networks extend beyond the prefrontal areas to the parietal, cerebellar, and subcortical areas [12]. It is also essential to explore whether these neural connections are abnormal in depression. In the study of Vasic et al., the functional connection of DLPFC and cingulate networks was assessed with fMRI and independent component analysis [13]. In this experiment, the Sternberg Item Recognition Paradigm was modified. During the stimulus period, three letters appeared on the screen for $1500 \mathrm{~ms}$. During a $600 \mathrm{~ms}$ delay, participants were demanded to focus only on those highlighted letters and remember the followed letters. During the $200 \mathrm{~ms}$ probe period, the subject indicated lowercase letters belonged to previously manipulated letters. The high latency of the WM task was significantly correlated with the abnormal connection of the DLPFC. Besides, the MDD patient group was found with an increased connection between DLPFC and the cerebellum compared with healthy controls. The healthy controls group in ACC was more associated with the ventrolateral side than the MDD patient group. Therefore, in the process of WM in MDD patients, the functional connectivity of brain regions is also abnormal. 


\section{RELEVANT INTERVENTIONS FOR DEPRESSION}

The activity in the bilateral prefrontal cortex area is what we mainly investigated to measure depression symptoms. Researchers often assess it through a series of working memory tasks because working memory impairment is an important manifestation of major depression [11]. The prefrontal cortex, especially the dorsolateral prefrontal cortex (DLPFC), is responsible for working memory to process and is associated with aggravation of MD symptoms. We reviewed relevant working memory interventions for depression as the following two levels: cognitive and neural.

Cognitive training is one of the fundamental approaches when it comes to the clinical treatment of depression. Cognitive control training (CCT) is a psychiatric treatment for depression. Its operating principle is to stimulate the cognitive activities in the DLPFC region to improve the control for increased amygdala activity and experience of stress. CCT includes many forms of interventions, one of the CCT versions lass and colleagues focused on is the paced auditory serial addition task (aPASAT). This cognitive task requires participants to add and sum the most recent numbers that have been presented to them from a stream of numbers. aPASAT aims to practice individuals' cognitive control ability over hyperactive amygdala [14]. Current work suggested that distress tolerance (DT) is negatively correlated with rumination. In contrast, rumination and depression symptoms are positively correlated, which means an increase in DT could reduce both rumination and depression symptoms. Lass and colleagues investigated if CCT, especially aPASAT, could achieve a chain reaction among those correlations mainly by enhancing the Dt. In their experiment, beck depression inventory (BDI) and the cage, a measure of problematic alcohol abuse, are adapted to evaluate depressive symptoms. Dt is recorded in the form of a survey in terms of how people respond to distress. The result of this experiment supports that the more distressing the cognitive tasks are, the more depressive symptoms participants manifest in a controlled condition. To compare it with the aPASAT group, the DT level didn't decrease. At the same time, the task's difficulty was constant, which indicates CCT (aPASAT)is effective and adaptive in enhancing distressing tolerance and decreasing rumination and depression symptoms.

While CCT effectively reduces depression symptoms, neurofeedback (NF)represents the potential effectiveness of neurological treatment techniques on depression. Neurofeedback training focuses on enhancing target peak alpha frequency (PAF) activation, the brain waves in the prefrontal region, to achieve an increase in cognitive performance. Such training in cognitive performance could bring changes in depression symptoms and rumination. According to $\mathrm{Yu}$ et al. (2020), rumination is a maladaptive emotional state that could aggravate depression symptoms [15]. In their experiment, Yu and colleagues assessed how the electroencephalogram neurofeedback protocol (NF) promotes cognitive preparedness in the prefrontal cortex and its effect on depression and rumination. In this study, the Chinese response style questionnaire and the Chinese version of the beck depression inventory-II (BDI-II) were adapted to evaluate depression symptoms and rumination tendency. Individual cognitive preparedness and performance are indicated by PAF: the brain wave index that indicates the activation of the prefrontal cortex area. Participants in the experimental group received neurofeedback training (NF) which involves visual and auditory cognitive feedback. The executive function (EF) is responsible for emotion regulation and was evaluated in this experiment using cognitive control and exercise training as well. The enhancement of target PAF activation through the NF protocol's effect is correlated with the increase in EF performance. The EF performance has a positive correlation with changes in both depression and rumination. The better the EF performance is, the more the changes in depression symptoms and rumination levels are. The result indicates that the NF protocol's effect increases emotion regulation and cognitive performance. Participants in the neurofeedback group have a reduction in depression symptoms and rumination through intervention in neuroresponse on emotion-relevant tasks.

Moreover, physical intervention is also feasible besides psychiatric (e.g.: CT), and neurological (e.g.: NF) treatment approaches. Transcranial direct current stimulation (tDCS) is a clinical treatment technique that uses subtle neuro stimuli on the scalp to make shifts in membrane resting potentials to modulate cortical excitability and cognitive function. Salehinejad and colleagues investigated whether tDCS in DLPFC (part of the brain that is associated with cognitive and executive function) could enhance visual working memory and reduce depression symptoms in MD patients [16]. The Beck Depression Inventory (BDI) and Hamilton Depression Scale (HDRS) were adapted to measure participants' depression symptoms in this experiment. For the tDCS session group, the anodal electrode was placed over the left DLPFC, and the cathode electrode was placed over the right DLPFC. In the tDCS active group, participants received a stimulation session each day for 10 consecutive days. Besides, their cognitive functions and brain dysfunctions in the frontal brain region were assessed using the Cambridge Neuropsychological Test Automated Battery (CANTAB). And the PRM test is used to measure visual recognition memory. Participants received a series of visual memory tasks to measure their cognitive functioning since visual memory is the most impaired cognitive domain in MD patients [16]. The result suggests that modulation in PFC brain activity reduces depression symptoms. In addition, 
anodal and cathodal tDCS both improve visual recognition memory in the tDCS active group. To sum up, tDCS over DLPFC improves impaired cognitive functioning and reduces depression symptoms.

\section{LIMITATION AND FUTURE DIRECTION}

Most of the current research on major depression and treatment development adapted the cross-sectional format. However, the etiology of depression and updating of brain cognitive stimuli are far more complicated and couldn't be comprehensively interpreted through only comparisons on a single developmental stage. Comparatively, future research needs to investigate from a more dynamic and vertical perspective on MD treatment development. The longitudinal experiment is a more exhaustive direction that researchers could adapt in the future to develop the most suitable intervention time and method for depression patients through observing behavioural and cognitive function at different ages and developmental stages. Second, current work often brings on an experiment in the context of a research lab. In this case, participants are often consciously aware that their cognitive ability was tested. It is intriguing and crucial to start cognitive experiments in a more naturally occurring environment to see if there are any confounding factors when situations change. Third, research on depression symptoms often go through the direction of the assessment of working memory. However, working memory has often been evaluated as an independent variable, but the interaction between the prefrontal cortex and other parts of the brain has been largely underestimated. Changes in other brain regions could also contribute to the etiology of depression. The interaction between multiple brain regions is one of the future research directions on major depression and its relation to working memory deficits.

\section{CONCLUSION}

To conclude, as far as emotional processing and working memory deficits in depression, there are maladaptive and protective biases in working memory; these biases may be the basis of the ability to effectively regulate negative emotions. Furthermore, depression was linked to difficulty removing extraneous negative information from working memory, and increased interference from extraneous negative material was related to rumination. In addition, individual differences in using emotion regulation strategies play a significant role in the onset of depression, and cognitive control deficits may impact the use of emotion regulation strategies. In terms of neural mechanisms, rumination was associated with deficits in cognitive control. Also, the function of the frontotemporal circuit may be abnormal during working memory processing in patients with MDD. Abnormal functional connectivity in brain regions during WM in MDD patients may be abnormal comparison healthy control. At the cognitive level, aPASAT is effective in reducing rumination. At neural level, neural feedback and tDCS are effective. One of the limitations of existing studies is that most studies of working memory defects using cross-sectional studies, comparing the single development stage. Another limitation is that many tasks used cognitive tasks. Furthermore, existing studies regarding working memory defect as a single factor, without considering its relationship with other factors. Future researchers need to examine the dynamic development stage in depression and focus on using tasks relied on a more naturally occurring environment. Also, considering the interaction of working memory between multiple brain regions is one of the future directions on depression.

\section{REFERENCES}

[1] Chai, W. J., Abd Hamid, A. I., \& Abdullah, J. M. (2018). Working Memory From the Psychological and Neurosciences Perspectives: A Review. Frontiers in Psychology, 9.

[2] Joormann, J., \& Gotlib, I. H. (2010). Emotion regulation in depression: Relation to cognitive inhibition. Cognition \& Emotion, 24(2), 281-298.

[3] Hagen, E. H. (2011). Evolutionary Theories of Depression: A Critical Review. The Canadian Journal of Psychiatry, 56(12), 716-726.

[4] Kupferberg, A., Hager, O. M., Fischbacher, U., Brändle, L. S., Haynes, M., \& Hasler, G. (2016). Testing the social competition hypothesis of depression using a simple economic game. BJPsych Open, 2(2), 163-169.

[5] Liu, R. T., Kleiman, E. M., Nestor, B. A., \& Cheek, S. M. (2015). The Hopelessness Theory of Depression: A Quarter-Century in Review. Clinical Psychology: Science and Practice, 22(4), 345-365.

[6] Levens, S. M., \& Gotlib, I. H. (2010). Updating positive and negative stimuli in working memory in depression. Journal of Experimental Psychology: General, 139(4), 654-664.

[7] Joormann, J., \& Gotlib, I. H. (2008). Updating the contents of working memory in depression: Interference from irrelevant negative material. Journal of Abnormal Psychology, 117(1), 182-192.

[8] Nolen-Hoeksema, S., Wisco, B., \& Lyubomirsky, S. (2008). Rethinking rumination. Perspectives on Psychological Science, 3, 400-424

[9] Joormann, J., Levens, S. M., \& Gotlib, I. H. (2011). Sticky Thoughts. Psychological Science, 22(8), 979-983. 
[10] Bunge SA, Klingberg T, Jacobsen RB, Gabrieli JD (2000). A resource model of the neural basis of executive working memory. Proceedings of the National Academy of Sciences USA 97, 3573-3578.

[11] Matsuo, K., Glahn, D. C., Peluso, M. A. M., Hatch, J. P., Monkul, E. S., Najt, P., ... \& Soares, J. C. (2007). Prefrontal hyperactivation during working memory task in untreated individuals with major depressive disorder. Molecular psychiatry, 12(2), 158-166.

[12] Wolf RC, Walter H (2005). Evaluation of a novel event-related parametric fMRI paradigm investigating prefrontal function. Psychiatry Research 140, 73-83.

[13] Vasic, N., Walter, H., Sambataro, F., \& Wolf, R. C. (2008). Aberrant functional connectivity of dorsolateral prefrontal and cingulate networks in patients with major depression during working memory processing. Psychological Medicine, 39(6), 977-987.

[14] Lass, A. N., Rokke, P. D., \& Winer, E. S. (2021). Evaluating cognitive control training on symptoms of depression over time: Three potential mechanisms. Journal of Affective Disorders Reports, 4, 100127.

[15] Yu, S. H., Tseng, C. Y., \& Lin, W. L. (2020). A Neurofeedback Protocol for Executive Function to Reduce Depression and Rumination: A Controlled Study. Clinical Psychopharmacology and Neuroscience, 18(3), 375.

[16] Salehinejad, M. A., Rostami, R., \& Ghanavati, E. (2015). Transcranial direct current stimulation of dorsolateral prefrontal cortex of major depression: improving visual working memory, reducing depressive symptoms. NeuroRegulation, 2(1), 3737. 